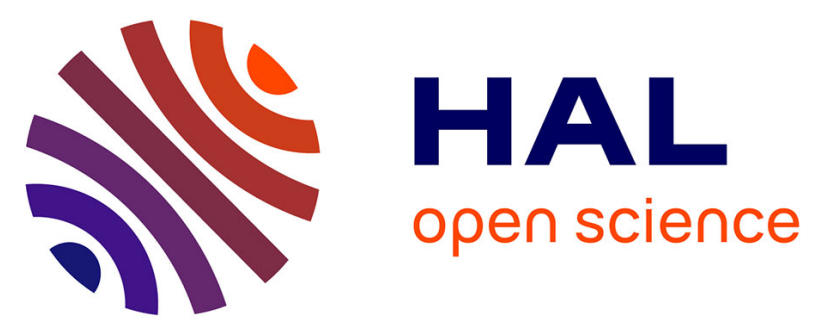

\title{
A prospective comparison of dynamic contrast-enhanced MRI and 51Cr-EDTA clearance for glomerular filtration rate measurement in 42 kidney transplant recipients
} Benjamin Taton, Renaud de La Faille, Julien Asselineau, Paul Perez, Pierre Merville, Thierry Colin, Christian Combe, Steven Sourbron, Nicolas Grenier

\section{To cite this version:}

Benjamin Taton, Renaud de La Faille, Julien Asselineau, Paul Perez, Pierre Merville, et al.. A prospective comparison of dynamic contrast-enhanced MRI and 51Cr-EDTA clearance for glomerular filtration rate measurement in 42 kidney transplant recipients. European Journal of Radiology, 2019, 117, pp.209-215. 10.1016/j.ejrad.2019.02.002 . hal-02416091

\section{HAL Id: hal-02416091 \\ https://hal.inria.fr/hal-02416091}

Submitted on 17 Dec 2019

HAL is a multi-disciplinary open access archive for the deposit and dissemination of scientific research documents, whether they are published or not. The documents may come from teaching and research institutions in France or abroad, or from public or private research centers.
L'archive ouverte pluridisciplinaire HAL, est destinée au dépôt et à la diffusion de documents scientifiques de niveau recherche, publiés ou non, émanant des établissements d'enseignement et de recherche français ou étrangers, des laboratoires publics ou privés. 


\section{A prospective comparison of DCE-MRI and ${ }^{51} \mathrm{Cr}$-EDTA for glomerular filtration rate measurement in 42 kidney transplant recipients}

Benjamin Taton (PhD, MD) $)^{1,2,5}$

Renaud De La Faille (MD) ${ }^{1}$

Julien Asselineau (MSc) ${ }^{3}$

Paul Perez (MD, PhD) ${ }^{3}$

Pierre Merville (MD, PhD) ${ }^{1}$

Thierry Colin $(\mathrm{PhD})^{2,4,5}$

Christian Combe (MD, PhD) ${ }^{1,6}$

Steven Sourbron (PhD) ${ }^{7}$

Nicolas Grenier (MD, PhD) ${ }^{8}$

${ }^{1}$ Centre hospitalier universitaire de Bordeaux, Service de néphrologie, transplantation, dialyse - Groupe hospitalier Pellegrin, France.

${ }^{2}$ Univ. Bordeaux, IMB, UMR CNRS 5251, F-33400, Talence, France.

${ }^{3}$ Centre hospitalier universitaire de Bordeaux, pôle de santé publique, unité de soutien méthodologique à la recherche clinique et épidémiologique, France.

${ }^{4}$ Bordeaux INP, IMB, FF-33400 Talence, France.

${ }^{5}$ INRIA Bordeaux Sud-Ouest, MONC team, FF-33400, Talence, France.

${ }^{6}$ Univ. Bordeaux, Unité INSERM 1026, France.

${ }^{7}$ Division of medical physics, University of Leeds, United Kingdom.

${ }^{8}$ Centre hospitalier universitaire de Bordeaux, Service de radiologie et d'imagerie diagnostique et interventionnelle de l'adulte, Groupe hospitalier Pellegrin, France.

Corresponding author:

Benjamin Taton

E-mail: benjamin.taton@chu-bordeaux.fr

Phone: 33556795538

Fax : 33556796107 


\title{
A prospective comparison of dynamic contrast- enhanced MRI and ${ }^{51} \mathrm{Cr}$-EDTA clearance for glomerular filtration rate measurement in 42 kidney transplant recipients
}

\begin{abstract}
Objectives:

To evaluate the performance of dynamic contrast-enhanced MRI measurement of glomerular filtration rate (GFR) compared with the reference standard technique of urinary clearance of ${ }^{51} \mathrm{Cr}$-EDTA.
\end{abstract}

\section{Patients and methods:}

All kidney transplant recipients (KTRs) with an indication for non-urgent contrastenhanced MRI at our institution were prospectively included between 2008 and 2012 . Renographies were acquired by low-dose DCE-MRI then fitted with a two-compartment pharmacokinetic model. MR-GFR was compared with reference isotopic measurements using Bland-Altman diagrams, intraclass correlation coefficient (ICC) and concordance rates.

\section{Results:}

Forty-two KTRs (mean age 51.5 years, 26 - 74) were analyzed. Mean estimated GFR was $48.5 \pm 27 \mathrm{~mL} / \mathrm{min} / 1.73 \mathrm{~m} 2(24-178 \mathrm{~mL} / \mathrm{min})$. The mean bias was $+13.2 \mathrm{~mL} / \mathrm{min}(6.4-$ $20.0,+36.9 \%)$ ranging from $-31.0 \mathrm{~mL} / \mathrm{min}(-41.7 \%)$ to $+101.4 \mathrm{~mL} / \mathrm{min}(+89.2 \%)$ with a 
large variability (standard-deviation: $22.3 \mathrm{~mL} / \mathrm{min}$; limits of agreement: [-30.6 (-43.3-$18.9) ;+57.0(45.3-68.7)])$. The ICC was $0.32(0.02-0.56)$ and the concordance rate was $28.6 \%(14.9-42.2)$.

\section{Conclusions:}

The large variability of MR-GFR compared with the reference technique precludes its use in KTRs, whose anatomical peculiarities make standardization of arterial input function (AIF) difficult.

Keywords: functional MRI; kidney transplant; glomerular filtration rate; data accuracy. 


\section{Abbreviations:}
AIF: arterial input function
DCE-MRI: dynamic contrast-enhanced MRI

Gd-CM: gadolinium based contrast media

GFR: glomerular filtration rate

KTR: kidney transplant recipients 


\section{Introduction}

GFR is the hallmark of kidney function in clinical practice. It is generally estimated using formulas that reflect the balance between endogenous synthesis and renal elimination of biological markers (namely creatinine and/or cystatin C) (1). These formulas were built by regression in large specific-population samples. As such, their use to estimate a specific individual's kidney function is often problematic. Measuring the clearance of exogenous markers infused into a patient's bloodstream is considered to be the gold standard for GFR measurement. However, these techniques are not well suited for routine evaluation of kidney function because they are either costly and cumbersome or rely on hypotheses that cannot always be justified. In addition, most often they require nuclear medicine services.

Gadolinium-based contrast media ( $\mathrm{Gd}-\mathrm{CM}$ ) have an excellent renal safety profile even in patients with impaired kidney function (2), and have the same pharmacokinetics as the tracers used for clearance measurement techniques (3). Dynamic contrast-enhanced MRI (DCE-MRI) monitors the distribution of Gd-CM in anatomic structures. In association with mathematical models that describe this process, these imaging techniques are promising tools to evaluate kidney function and other physiological parameters of potential interest in nephrology (e.g. renal blood flow, and vascular or tubular transit times). Compared with isotopic methods, MRI provides high-quality anatomic descriptions of the studied organs and as such, it could provide functional maps of native and transplanted kidneys.

Many studies have found encouraging results for native kidneys, in both healthy or diseased (4-11) subjects but biases were highly dependent on both the acquisition 
protocol and the model used, and error variability was excessively large. Actually, only Lim (11) achieved performances compatible with a clinical use of the technique.

To our knowledge, the case of KTRs has been studied only by Yamamoto et al. (12). These authors focused on the diagnostic value of tubular transit times for acute rejection, but did not compare MR-GFR with a reference measurement. Investigation of KTRs offers a rewarding clinical study group because technically they show only slight respiratory movements, and clinically their follow-up often implies iterative graft biopsies, making non-invasive procedures highly worthwhile. Moreover, most of them present an impaired kidney function, and filtration is almost completely performed by the kidney allograft so that there is no need to determine differential filtration to compare MR-GFR with reference GFR estimations. This is the first study whose aim was to compare the performances of DCE-MRI GFR measurements with ${ }^{51} \mathrm{Cr}$-EDTA clearance as a reference technique in KTRs.

\section{Materials and methods}

\subsection{Patients}

This prospective study was approved by the institutional review board and the interregional ethics authorities (Comité de protection des personnes Sud-Ouest et Outre-Mer III), and informed written consent was obtained from all patients. Between January 2008 and January 2012, all patients with renal transplantation followed in our department, whose medical condition required a non-urgent contrast-enhanced MRI of the renal graft, and who had an estimated GFR over $20 \mathrm{~mL} / \mathrm{min} / 1.73 \mathrm{~m}^{2}$ according to the 
MDRD formula (13), were considered for inclusion to undergo a low-dose MR renography.

Patients with contraindications to MR examinations or isotopic determinations of the GFR were not included in the study (pregnant or breast-feeding women, patients with implanted electronic devices, metallic foreign bodies or surgical clips, severe claustrophobia, known intolerance or allergy to $\mathrm{Gd}-\mathrm{CM}$ ).

Demographic data was gathered from the patients' medical records and from electronic databases. A blood sample was taken to measure creatininemia and hematocrit. Isotopic GFR measurement and DCE-MRI examination were performed on the same day to avoid any change in kidney function between measurements.

\subsection{Magnetic resonance imaging}

MRI images were acquired on a 1.5T MRI scanner (ACS-NT - Philips) using a body phased-array coil. A three-dimensional saturation-recovery turbo-field echo sequence was used with the following parameters: $\operatorname{TE} / \operatorname{TR}=3.7 / 6.2 \mathrm{~ms} ; \theta=10^{\circ} ;$ slice thickness $=$ $10 \mathrm{~mm}$, no gap; 5 slices; acquisition matrix $60 \times 240$; reconstructed matrix $256 \times 256$; approximate voxel size: $1.6 \times 1.6 \times 10 \mathrm{~mm}^{3}$; parallel imaging (SENSE method, 1.7 reduction factor). The saturation pulse was applied non-selectively to avoid inflow effects within the volume. A coronal oblique section was selected to include both the entire kidney allograft on its long axis and the terminal abdominal aorta within the acquisition volume, and centered on the renal pedicle. However ${ }_{2}$ in difficult cases, kidney parenchyma was given priority over the terminal aorta, provided that an arterial signal remained visible in the acquisition volume. 
The temporal resolution of the sequence was approximately 2 seconds. Before and after injection of the contrast agent, images were acquired iteratively 200 times across 6 min $40 \mathrm{~s}$ without breath holds; the patient was simply asked to breath slowly. As of the 20th acquisition, each patient received an intravenous injection of $0.07 \mathrm{~mL} / \mathrm{kg}(33 \%$ of a standard dose) of gadoterate-meglubine (Dotarem@; Guerbet, Roissy, France) with an infusion rate of $2 \mathrm{~mL} / \mathrm{s}$, followed by a $20 \mathrm{~mL}$ saline flush at $2 \mathrm{~mL} / \mathrm{s}$.

In addition to the functional sequence, all subjects underwent standard T1-weighted gradient echo and T2-weighted fast spin-echo imaging, and 3D contrast-enhanced MR angiography for morphologic assessment.

\subsubsection{Data analysis}

\section{Image processing}

Area under the Gd-CM concentration curve (AUC) was computed for each voxel of the functional acquisition. For each patient, a threshold was manually chosen to identify a small subset of voxels with the highest AUC in the aorta or the common iliac artery. This lead to select a region in the center of the terminal aorta, 2-3 pixels away from aortic boundaries. Quite often, the anatomical configuration made it impossible to acquire both the graft and the terminal aorta in the same data volume. In such cases, the arterial region of interest (ROI) was selected in the common iliac artery or in the upper aorta, depending on the place where the highest AUC were found. The AUC image was also used to manually delineate the kidney parenchyma (pelvis excluded) on each of the five slices available for each patient. Motion of the kidney during the acquisition was ignored. Examples of typical segmentations are shown on Fig. 1. 
The arterial and renal signals were averaged over the corresponding ROI before being used as input for the model-fitting algorithm. Signals corresponding to the images and segmentations given on Fig. 1 are presented as examples on Fig. 2. Kidney volume $(V)$ was computed directly from these ROI as the product of a voxel volume by the number of voxels in the selected region.

Image manipulations and delineations were performed offline using a program developed by (initials) using PMI (v. 0.4) and written in IDL (v 6.3).

\section{Compartment model}

The distribution of Gd-CM in the kidney was described using the compartmental model proposed by Sourbron et al. (9) and depicted on Fig. 3.

Gadolinium concentration was assumed to be proportional to the increase of the signal intensity from the basal situation, denoted $s_{0}$, which was computed from the 20 first images: $\quad c(t) \simeq k \times\left(s(t)-s_{0}\right)$. Coefficient $k$ is unknown but cancels out in further computations so that it does not need estimating. The plasma concentration of gadolinium in the aorta was computed from full blood concentration by correcting for the hematocrit when available. For eleven patients it was not known and was replaced with the mean value over the whole cohort (35.5\%).

The 4 parameters of the model (renal plasma flow, GFR, plasma volume relative to the kidney volume, tubular mean transit time) were determined by fitting the predicted tissue concentration with measured data (likelihood maximization using the LevenbergMarquardt algorithm (14)). The convergence of the optimization algorithm to a plausible solution was checked visually by comparing the fitted curve with actual data. 
Computations were implemented in Python and its associated scientific computing libraries (15).

\subsubsection{Isotopic GFR measurement}

Reference GFR values were obtained by measurements of ${ }^{51} \mathrm{Cr}$-EDTA renal clearance (16). A bolus of $100 \mu \mathrm{Ci}(3.7 \mathrm{MBq}){ }^{51} \mathrm{Cr}-\mathrm{EDTA}$ was injected at $\mathrm{t}=0$. Each patient was asked to drink $5 \mathrm{~mL} / \mathrm{kg}$ of water at the beginning of the examination and $90 \mathrm{~mL}$ at $\mathrm{t}=60$ min and asked to void at $\mathrm{t}=60 \mathrm{~min}$. Blood samples were taken at $t=75,105,135$ and 165 min to determine the plasma concentrations of ${ }^{51} \mathrm{Cr}$-EDTA $\left(P_{t}\right)$. Patients were asked to void at $\mathrm{t}=90,120,150$ and $180 \mathrm{~min}$ and to drink $90 \mathrm{~mL}$ water at each of these time point. The volume of urine and urine concentrations of ${ }^{51} \mathrm{Cr}$-EDTA were determined for each of these samples $\left(V_{t_{1}-t_{2}}, U_{t_{1}-t_{2}}\right)$.

The GFR was determined as the mean of four calculations of the urinary clearance of ${ }^{51} \mathrm{Cr}$ - EDTA for each time point:

$G F R=\frac{1}{4}\left(\frac{U_{60-90} \times V_{60-90}}{P_{75}}+\frac{U_{90-120} \times V_{90-120}}{P_{105}}+\frac{U_{120-150} \times V_{120-150}}{P_{135}}+\frac{U_{150-180} \times V_{150-180}}{P_{165}}\right)$

An expert (initials) reviewed all these measurements. Patients showing significant deviations from this protocol or with large discrepancies between the four clearance measurements (coefficient of variation over 10\%) were excluded from the study.

\subsubsection{Statistics}

MR-GFR and ${ }^{51} \mathrm{Cr}-\mathrm{EDTA}-\mathrm{GFR}$ were compared using Bland-Altman diagrams (17-19), intra-class correlation coefficients (ICC) (20) and concordance rates (namely, the proportion of patients whose GFR measurements did not differ by more than $5 \mathrm{~mL} / \mathrm{min}$ 
between the two techniques). Linear regression and correlation coefficients were given for comparison with previous works. Normality of error distribution in the Bland-Altman analysis was tested using Kolmogorov-Smirnov tests.

As we expected an ICC greater than 0.8 , we calculated the minimum sample size to be 55 to obtain a lower bound of the $95 \%$ confidence interval of at least 0.6 (this threshold is considered to represent good agreement between the investigated techniques) (21).

Demographic data are presented as mean \pm standard-deviation or median [first; third quartile] when appropriate. Comparisons of GFR measurement error between subgroups were performed using Wilcoxon tests. Subgroups were defined depending on the immunosuppressive regimen, the indication of MRI examinations, and the abnormalities reported by the radiologist who interpreted the standard morphological acquisitions.

Statistics were computed using the R software (version 3.1.2) and the corresponding packages $(22,23)$.

\section{Results}

Patient selection is shown in the flow diagram in Fig. 4. Sixty-nine patients were initially included in the study. Twenty-seven were excluded because MR renography was not interpretable (MRI artefacts or poor positioning of the acquisition volume resulting in sequences without dependable arterial signal) (15 patients), or because their isotopicGFR calculation was untrustworthy (12 patients). Finally, 42 patients were analysed (29 men, 13 women; mean age 51.5 years; age range $26-74$ ) (Table 1). The median time 
from kidney transplantation to isotopic measurements and MRI examination was $397[113 ; 1145]$ days.

For most patients, acquiring both the entire kidney and the terminal abdominal aorta at the same time proved impossible and arterial ROI had to be selected in the upper aorta or in the common iliac artery: the ROI was taken in the aorta for 35/42 (83.3\%) patients, and in the iliac artery for $7 / 42(16.7 \%)$ patients. The size of the arterial ROI was on average $62 \pm 28$ voxels (median: 54.5, range: $23-154$ ) for the aortic region, and $8878 \pm 2318$ voxels (median: 8089.5 , range: $5617-15262$ ) for the kidney parenchyma (average volume of the kidney: $203 \pm 50 \mathrm{~mL}$; median: 192; range: $135-321$ ).

Mean estimated GFR (MDRD formula) of our patients was $48.5 \pm 27 \mathrm{~mL} / \mathrm{min} / 1.73 \mathrm{~m}^{2}$ (eGFR range: from 24 to 178). Mean GFR measured by the isotopic reference technique was $41.8 \pm 14.5 \mathrm{~mL} / \mathrm{min}$ (EDTA-GFR range: from 18.3 to 81.1 ). Mean GFR measured by DCE-MRI was $55.0 \pm 26.0 \mathrm{~mL} / \mathrm{min}$ (MR-GFR range: from 23.9 to $170.1 \mathrm{~mL} / \mathrm{min}$ ). As plasma samples were available, we also determined the plasma clearance of ${ }^{51} \mathrm{Cr}$-EDTA according to Bröchner-Mortensen's technique (24) as an alternative reference measurement. As already stated in previous works (25), the two techniques were in good agreement, plasma clearance being slightly higher that renal clearance (mean difference between measurements: $4.3 \pm 7.6 \mathrm{~mL} / \mathrm{min}$ ). The use of either reference technique did not change the conclusion of our study (see supplemental material Fig. S3 and S4).

The comparison between MR-GFR and the reference method is depicted in Fig. 5 . There was a fair correlation between both measurements $(p<0.001, r=0.52)$. The 
regression line of MR-GFR against EDTA-GFR had a slope of 0.92 and an intercept of $16.5 \mathrm{~mL} / \mathrm{min}$. Our measurement protocol lead to a large overestimation of the GFR compared with the reference technique. The mean difference with the reference technique was $+13.2 \pm 22.3 \mathrm{~mL} / \mathrm{min}(6.4-20.0,+36.9 \%)$ with a large variability (limits of agreement: [-30.6(-42.3--18.9); 57.0(45.3-68.7)]). The ICC was $0.32(0.02-0.56)$, far below the 0.6 threshold for satisfactory agreement between the two techniques. The concordance rate was $28.6 \%(14.9-42.2)$. Finally, on average, the systematic bias was slightly increasing with the GFR value $(+0.28 \mathrm{~mL} / \mathrm{min}$ per $\mathrm{mL} / \mathrm{min}$ increase).

When comparing subgroups of patients depending on their immunosuppressive regimen, the indication for the $\mathrm{MRI}$, or the morphological abnormalities, no specific characteristic presented a significant association with larger measurement errors (Fig. 6).

To investigate the influence of $\mathrm{ROI}$ selection on measured GFR, we restricted our analysis to the patients for whom the AIF could be determined from the aorta (36/42, $86 \%$ of patients). In these patients, the mean bias was $11.6 \pm 18.3 \mathrm{~mL} / \mathrm{min}$, with [-24.2(-34.6-13.8); +47.6(37-57.8)] limits of agreement (vs.13.2 $\pm 22.3 \mathrm{~mL} / \mathrm{min}$ in the whole cohort). The decrease in error variability was not statistically significant $(p=0.38$ for the modified one-sided paired Pitman-Morgan test). In a second experiment the AIF was determined from the iliac artery in a region as close as possible of the implantation of the kidney allograft artery and the GFR was computed using this new AIF (this was possible for $37 / 42$ (88\%) patients). In comparison with the aortic AIF, the mean bias was $24.2 \pm 25.5 \mathrm{~mL} / \mathrm{min}$ (vs. $11.6 \pm 18.3$ ) with $[-25.8(-40.3--11.3) ;+74.3(59.8-88.8)]$ limits of agreement. The decrease in error variability did not reach statistical significance 
$(p=0.26)$. The associated Bland-Altman diagrams are presented in the supplemental material (Fig. S2).

\section{Discussion}

This is the first study performed in a cohort of KTRs for whom non-invasive GFR measurement would be extremely worthwhile and who show a wide range of GFR values measured with a reference technique. We chose to exclude all the patients with doubtful isotopic measurements (12/69) to reinforce the value of this reference technique, keeping only trustworthy results.

Overall, while using DCE-MRI to estimate GFR was feasible for KTRs, compared to the reference technique, DCE-MRI strongly overestimated GFR and exhibited a large variability with poor intra-class correlation coefficients and low concordance rates.

Whereas there is no other experience in the literature on KTRs for comparison, our results are somewhat consistent with previously published work on native kidneys but exhibit a higher systematic bias and larger error variability.

Using a Rutland-Patlak technique in 28 diseased subjects, Hackstein et al. (5) found a correlation coefficient $r=0.86$ between iopromide clearance measurements and MRGFR, and a standard deviation from the regression line of $14.8 \mathrm{~mL} / \mathrm{min}$. In 39 patients with a large range of GFR, Buckley et al. (6) also found a strong correlation between isotopic reference measurements and MR-GFR (Spearman's $\rho: 0.81$ ). In another population of diseased subjects, using and slightly different pharmacokinetic models but the same acquisition protocol, Lee et al. (7) and Zhang et al. (8) obtained consistent 
results: mean bias of -11.8 and $-18.1 \mathrm{~mL} / \mathrm{min}$, and variability of \pm 13.7 and $\pm 13.9 \mathrm{~mL} / \mathrm{min}$ respectively in comparison with isotopic GFR determination (correlation coefficient was $r=0.82$ ). In the same population, with the same acquisition protocol and reference technique but with 8 different pharmacokinetic models, Bokacheva et al. (9) also found a good correlation between MR-GFR and reference measurements (correlation coefficients ranging from 0.74 to 0.85 ). Nonetheless, biases were highly dependent on the model used, ranging from $-52 \%$ to $-2.5 \%$. Vivier et al. (11) experimented other acquisition and post-treatment protocols with variants of the pharmacokinetic model by Zhang et al. in 20 patients with cirrhosis. Depending on the variant of the model and the orientation of the slice used for the post-processing, they found a median bias ranging from -7.7 to $-4.1 \mathrm{~mL} / \mathrm{min}$, with a root mean square error between 12.8 and $12.9 \mathrm{~mL} / \mathrm{min}$. The most promising results were obtained by Lim et al. in diseased patients with a wide range of GFR (12). Compared with reference isotopic GFR measurements, their protocol achieved a non-significant mean bias of $-0.7 \mathrm{~mL} / \mathrm{min}$ and variability of $\pm 5.86 \mathrm{~mL} / \mathrm{min}$, small enough to be compatible with clinical use.

Discrepancies of our results with previous works could be explained both by anatomic characteristics of transplanted kidneys compared to natives ones and by our workflow with respect to these characteristics.

In term of anatomic characteristics, in contrast with native kidneys, renal allografts exhibit a large variability in their anatomical configurations. This problem, which has been highly underestimated, made very difficult to combine an accurate positioning of the acquisition slab along the long axis of the graft and inclusion of the terminal aorta or of the common iliac artery. As illustrated on Fig. 2, this resulted in difficulties to achieve 
standardized and reproducible ROls selection for the AIF. The $10 \mathrm{~mm}$ thick coronal slices also favoured partial volume effects (PVE), mainly when AIF had to be sampled on iliac arteries instead of aorta, resulting in an underestimation of the AIF, and subsequently, in an overestimation of GFR. As the importance of PVE depends on the position of the acquisition matrix with respect to the arteries, which cannot be controlled, this probably accounts for a large part of the higher variability we noticed compared with measurements on native kidneys. The increase in the bias noticed when using an AIF sampled from the iliac arteries (see supplemental material, Fig. 4), which are more prone to PVE due to their smaller diameter, is consistent with this hypothesis. Finally, the close proximity of renal parenchyma with iliac vessels could also produce PVE, mixing signals coming from both structures.

Considering the model of Gd-CM pharmacokinetics, the interstitial compartment induces large overestimation of GFR. This hypothesis is consistent with the results obtained in most previous studies since the most negative biases are noticed mostly in the patients with the highest reference GFR measurements. In our cohort, most patients had an impaired kidney function, a setting often associated with fibrosis in KTRs, which could explain the observed positive bias. However, no histological evaluation of the interstitial volume was performed, and this hypothesis remains speculative.

Also, in our population of KTRs, the whole filtration function was attributed the transplant. However, some patients actually have a residual function from their native kidneys that presumably ranges from 0 to $10 \mathrm{~mL} / \mathrm{min}$. While this hypothesis is not consistent with GFR overestimation, it cannot be ruled out and may explain part of the large variability we noticed. 
At last, kidney motion was ignored because transplants are located far away from the diaphragm muscle. However spontaneous voluntary or digestive motions actually occurred and have inescapably increased error variability. This suggests that, even for KTRs, motion correction could prove beneficial to obtain reproducible results.

\section{Conclusion}

This first study on the performance of MR-measurement of GFR in KTRs with respect to a reference technique shows that, even if kidney grafts are unique, less mobile and more superficially located, an overestimation and a large variability still precludes its use in clinical practice without significant improvements. Anatomical constraints make the standardization of ROI selection more difficult than in native kidneys and lead to larger and unpredictable partial volume effects. These characteristics hamper an accurate and reproducible measurement of AIF and probably contribute for a large part to bias and variability.

\section{Acknowledgements}

Blinded for submission.

\section{References}

1. Levey A, Inker L, Coresh J. GFR estimation: from physiology to public health. Am J Kidney Dis. 2014;63:820-834.

2. Soyer P, Dohan A, Patkar D, Gottschalk A. Observational study on the safety profile of gadoterate meglumine in 35,499 patients: the SECURE study. J Magn Reson Imaging. 2017;45(4):988-997. 
3. Choyke P, Austin H, Frank J, Girton M, Diggs R, Dwyer A, et al. Hydrated clearance of gadolinium-DTPA as a measurement of glomerular filtration rate. Kid Int. 1992;41:1595-1598.

4. Hackstein N, Heckrodt J, Rau W. Measurement of single-kidney glomerular filtration rate using a contrast-enhanced dynamic gradient-echo sequence and the Rutland-Patlak plot technique. J Magn Reson Im. 2003;18:714-725.

5. Buckley D, Shurrab A, Cheung C, Jones A, Mamtora H, Kalra P. Measurement of single kidney function using dynamic contrast-enhanced MRI: comparison of two models in human subjects. J Mag Reson Imaging. 2006;1117-1123.

6. Lee V, Rusinek H, Bokacheva L, Huang A, Oesingmann N, Chen Q, et al. Renal function measurements from MR renography and a simplified multicompartment model. Am J Physiol Ren Physiol. 2007 May;292:F1548-F1559.

7. Zhang J, Rusinek H, Bokacheva L, Lerman L, Chen Q, Prince C, et al. Functional assessment of the kidney from magnetic resonance and computed tomography renography: impulse retention approach to a multicompartmental model. Magn Reson Med. 2008 Feb;59(2):278-288.

8. Bokacheva L, Rusinek $\mathrm{H}$, Zhang J, Chen Q, Lee V. Estimates of glomerular filtration rate from MR renography and tracer kinetics models. J Magn Reson Imaging. 2009;29:371-382.

9. Sourbron S, Michaely $\mathrm{H}$, Reiser M, SO S. MRI-measurement of perfusion and glomerular filtration in the human kidney with a separable compartment model. Invest Radiol. 2008 Jan;43(1):40-48.

10. Vivier P, Storey P, Rusinek H, Zhang J, Yamamoto A, Tantillo K, et al. Kidney function: glomerular filtration rage measurement with MR renography in patients with cirrhosis. Radiology. 2011;259(2):462-470.

11. Lim S, Chrysochou C, Buckley D, Kalra P, Sourbron S. Prediction and assessment of responses to renal artery revascularization with dynamic contrastenhanced magnetic resonance imaging: a pilot study. Am J Physiol Ren Physiol. 2013;305(5):F672-F678.

12. Yamamoto A, Zhang J, Rusinek $H$, Chandarana $H$, Vivier $P$, Babb J, et al. Quantitative evaluation of acute renal transplant dysfunction with low-dose three dimensional MR renography. Radiology. 2011;260(3):781-789.

13. Lenihan C, O'Kelly P, Mohan P, Little D, Walshe J, Kieran N, et al. MDRDestimated GFR at one year post-renal transplant is a predictor of long-term graft function. Ren Fail. 2008;30:345-352.

14. Press W, Teukolsky S, Vetterling W, Flannery B. Numerical recipes: the art of scientific computing. In Cambridge University Press; 2007. p. 773-839.

15. Jones E, Oliphant T, Peterson P, others. SciPy: Open source scientific tools for Python [Internet]. 2001. Available from: http://www.scipy.org/

16. Soveri I, Berg U, Björk J, Elinder C, Grubb A, Mejare I, et al. Measuring GFR: a systematic review. Am J Kid Dis. 2014;64(3):411-424. 
17. Bland J, Altman D. Statistical methods for assessing agreement between two methods of clinical measurement. Lancet. 1986 Feb;307-310.

18. Hamilton C, Stamey J. Using Bland-Altman to assess agreement between two medical devices - don't forget the confidence intervals! J Clin Monit Comput. 2007;21(6):331-333.

19. Euser A, Dekker F, le Cessie S. A practical approach to Bland-Altman plots and variation coefficients for log-transformed variables. J Clin Epidemiol. 2008;61(10):978982.

20. Bartko J. The intraclass correlation coefficient as a measure of reliability. Physiol Rep. 1966;19:3-11.

21. Bonett D. Sample size requirements for estimating intraclass correlations with desired precision. Stat Med. 2002;21:1331-1335.

22. Team RC. R: A language and environment for statistical computing [Internet]. Vienna, Austria: R Foundation for Statistical Computing; 2013. Available from: http://www.R-project.org

23. Gamer M, Lemon J, Fellows I, Singh P. irr: various coefficients of interrater reliability and agreement [Internet]. 2012. Available from: http://CRAN.Rproject.org/package=irr

24. Bröchner-Mortensen J. A simple method for the determination of glomerular filtration rate. Scand J Clin Lab Invest. 1972;30:271-274.

25. Moore A, Park-Holohan S, Blake G, Fogelman I. Conventional measurements of GFR using 51Cr-EDTA overestimate true renal clearance by 10 percent. Eur J Nucl Med. 2003;30(1):4-8.

Tab. 1. Demographic characteristics of the $\mathbf{4 2}$ kidney-transplant recipients and 34 donors analyzed. The number of patients for which the data were available is given in the third column $(n)$. 
Fig. 1. Examples of manual delineations of arterial and parenchymal region of interest (red regions) on the AUC images. Left: case where both the terminal aorta and the kidney allograft could be included in the same acquisition volume. Isotopic GFR was $34.4 \mathrm{~mL} / \mathrm{min}$, MR-GFR was $60.7 \mathrm{~mL} / \mathrm{min}$. Right: case where the anatomical configuration made this impossible. In this case, the distinction between the common iliac aorta and the allograft parenchyma is very difficult, due to anatomical proximity and partial volume effects (white arrows). Isotopic GFR was $81.1 \mathrm{~mL} / \mathrm{min}$, MR-GFR was 69.4 $\mathrm{mL} / \mathrm{min}$.

Fig. 2. Gadolinium concentration time curves in the blood (red, solid), the allograft parenchyma (black, dashed), and predicted by the model with the optimal parameters (purple, dash-dotted). The presented signals correspond to the mean value of the corresponding ROls, as presented on Fig. 1. Left (top), and Fig. 1. Right (bottom).

Fig. 3. Pharmacokinetic model used in the study. Gadolinium enters the vascular compartment (denoted $p$, with a volume $v_{p}$ ) with arterial plasma with a flow that corresponds to the renal plasmatic flow $(R P F)$ and a concentration $c_{A}(t)$. Part of it is filtered into a tubular compartment (denoted e, with a volume $v_{e}$ ) with a coefficient that corresponds to the glomerular filtration rate $(G F R)$. The remaining $(R P F-G F R)$ is returned to the general circulation. The filtered gadolinium is subsequently eliminated into the bladder with a transit time that is a parameter of the model. The dashed line represents reabsorption of gadolinium-free fluid.

Fig. 4. Flow-chart of the study. Twelve patients were excluded because their reference measurement was not reliable (large discrepancies between the four measurements of the renal clearance of ${ }^{51} \mathrm{Cr}$-EDTA). Fifteen patients were excluded because the MRI acquisition was not suitable for GFR measurements (MRI artifacts, bad positioning of the acquisition volume).

Fig. 5. Relationship between MR-GFR and the reference measurements. Top-left: linear regression of MR-GRF against ${ }^{51} \mathrm{Cr}$-EDTA clearance: slope was 0.92 , intercept was $16.5 \mathrm{~mL} / \mathrm{min}$, correlation coefficient was 0.52 . The regression line is plotted with a solid line. The (ideal) identity line is plotted with a dashed line. Each point corresponds to one the measurements for one patient. Top-right: Bland-Altman diagram. The dashed line represents the mean bias over the whole cohort $(+13.2 \mathrm{~mL} / \mathrm{min})$. Dotted lines represent the limits of agreement $([-30.6 ;+57.0])$. Normality of errors was tested using a Kolmogorov-Smirnov test $(p=0.21)$. The ideal no-difference line is draw with a solid line. Each point corresponds to the measurement for one patient. Bottom-left: BlandAltman analysis with log-transformed data (Kolmogorov-Smirnov test: $p=0.90$ ). Bottomright: limit of agreement computed from Bland-Altman analysis of the log-transformed data. On average, the systematic bias is slightly increasing with the GFR $(+0.28 \mathrm{~mL} / \mathrm{min}$ per $\mathrm{mL} / \mathrm{min}$ increase). The dashed line corresponds to the mean ratio between the bias and the mean of EDTA clearance and MR-GFR. The dotted lines correspond to the limit of agreements of the ratio, as depicted in the bottom-left figure. 
Fig. 6. Discrepancies between EDTA clearance and MR-GFR (relative values) depending on the use of calcineurin inhibitors in the patient's immunosuppressive regimen (Wilcoxon exact test: $p=0.32$ ), the indication of the MRI examinations (exact Wilcoxon tests: $p=0.08,0.68,0.99,0.42,0.84$ for hypertension, vascular anomaly, urological anomaly, renal mass and kidney failure respectively), and on the abnormalities reported by the radiologist (Wilcoxon exact test: $p=0.85,0.06,0.71$ and 0.08 for the association with renal artery stenosis, dilation of pelvi-caliceal cavities, perirenal collection and perfusion defect respectively). 


\section{Acknowledgements}

This study was supported by a public grant from the French National Research Agency (ANR) within the context of the "Investment for the Future" program, referenced ANR-10-LABX-57 and named TRAIL, the French State managed by the ANR referenced ANR-10-IDEX-03-02, named IdEx Bordeaux CPU, and the Centre Hospitalier Universitaire de Bordeaux (AOI-05). The authors thank Corinne Castermans, for her help in data managing and Pippa McKelvie-Sebileau, MSc, Bordeaux, France, for medical editorial assistance in English. 


\begin{tabular}{lcc}
\multicolumn{1}{c}{ Characteristics } & Value & $n$ \\
\hline Patient & $51.5 \pm 12.9$ & 42 \\
age $(\mathrm{yrs})$ & $29(69.1 \%) / 13(30.9 \%)$ & 42 \\
males / females & $48.5 \pm 27.0$ & 42 \\
eGFR $\left(\mathrm{mL} / \mathrm{min} / 1.73 \mathrm{~m}^{2}\right)^{*}$ & $35.5 \pm 5.3$ & 31 \\
hematocrit $(\%)$ & & \\
Kidney donor & $50.6 \pm 16.6$ & 34 \\
age (yrs) & $17(50 \%)$ & 34 \\
males & $17(50 \%)$ & 34 \\
females & $397[113 ; 1445]$ & 42 \\
Elapsed time from graft to MRI & $37(88.1 \%)$ & 42 \\
Immunosuppressive regimen & & \\
calcineurin inhibitors & $24(57.2 \%)$ & 42 \\
Indication for MRI examination & $8(19 \%)$ & 42 \\
vascular anomaly & $3(7.1 \%)$ & 42 \\
urologic anomaly & $2(4.8 \%)$ & 42 \\
arterial hypertension & $2(4.8 \%)$ & 42 \\
kidney failure & $3(7.1 \%)$ & 42 \\
renal mass & & \\
other & &
\end{tabular}

Tab. 1. Demographic characteristics of the 42 kidney-transplant recipients and 34 donors analyzed. The number of patients for which the data were available is given in the third column $(n)$. 


\section{Figure 1

Click here to download high resolution image

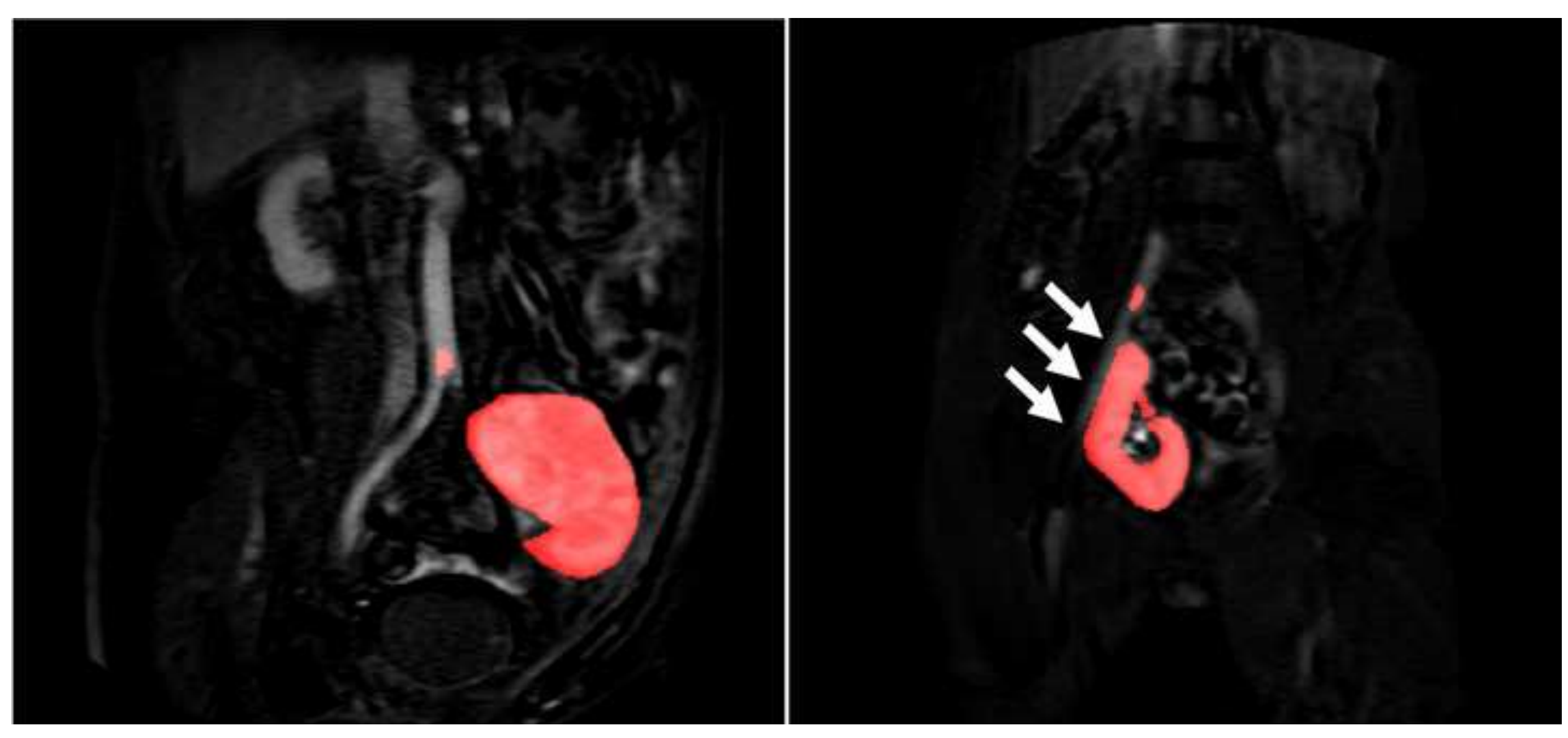



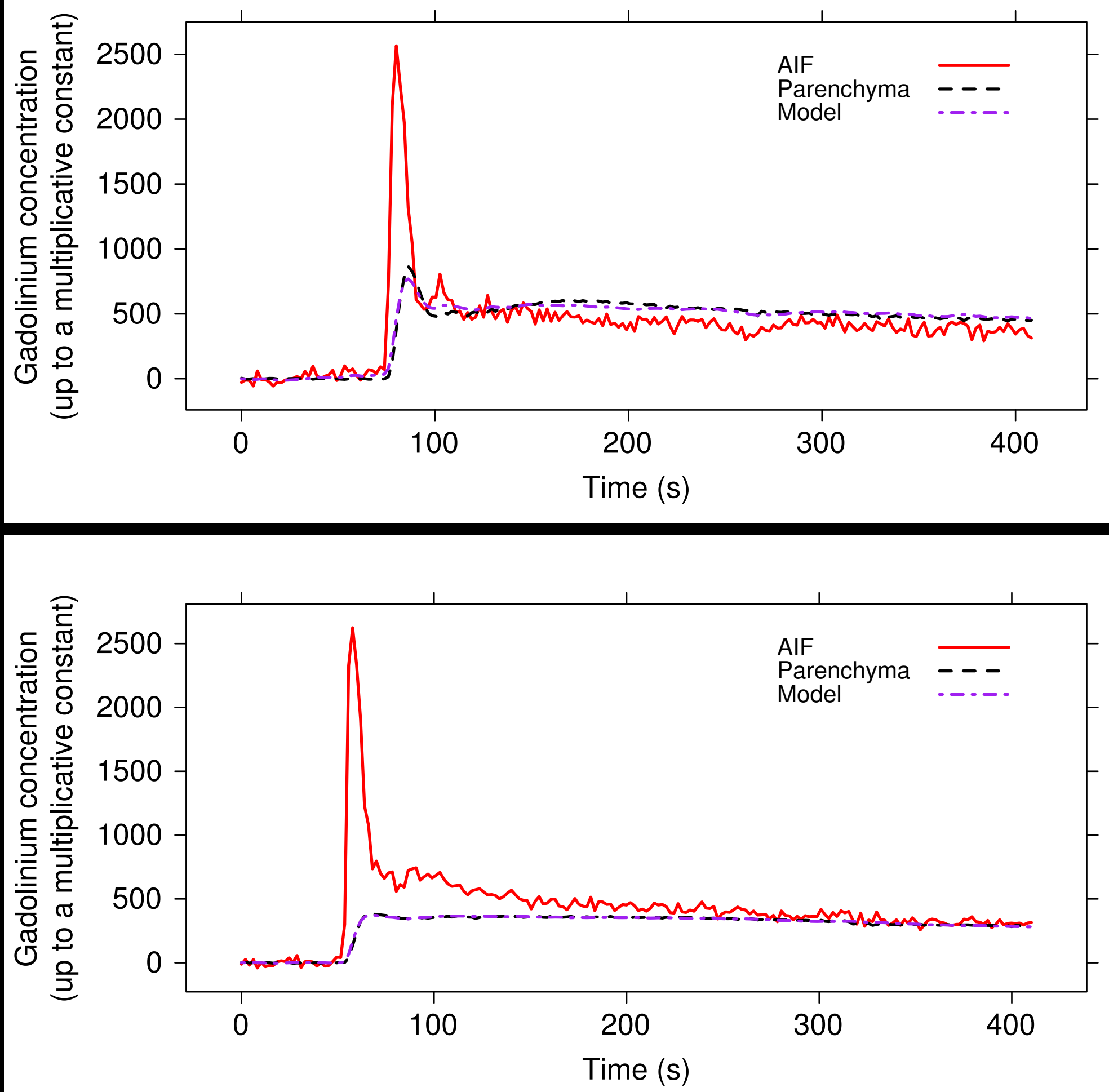
Figure 4

69 kidney transplant recipients included

\section{2 improper reference measurements}

\section{5 improper DCE-MRI examinations}

42 patients with both a valid ${ }^{51} \mathrm{Cr}$-EDTA clearance

measurement and a DCE-MRI

\section{examination.}



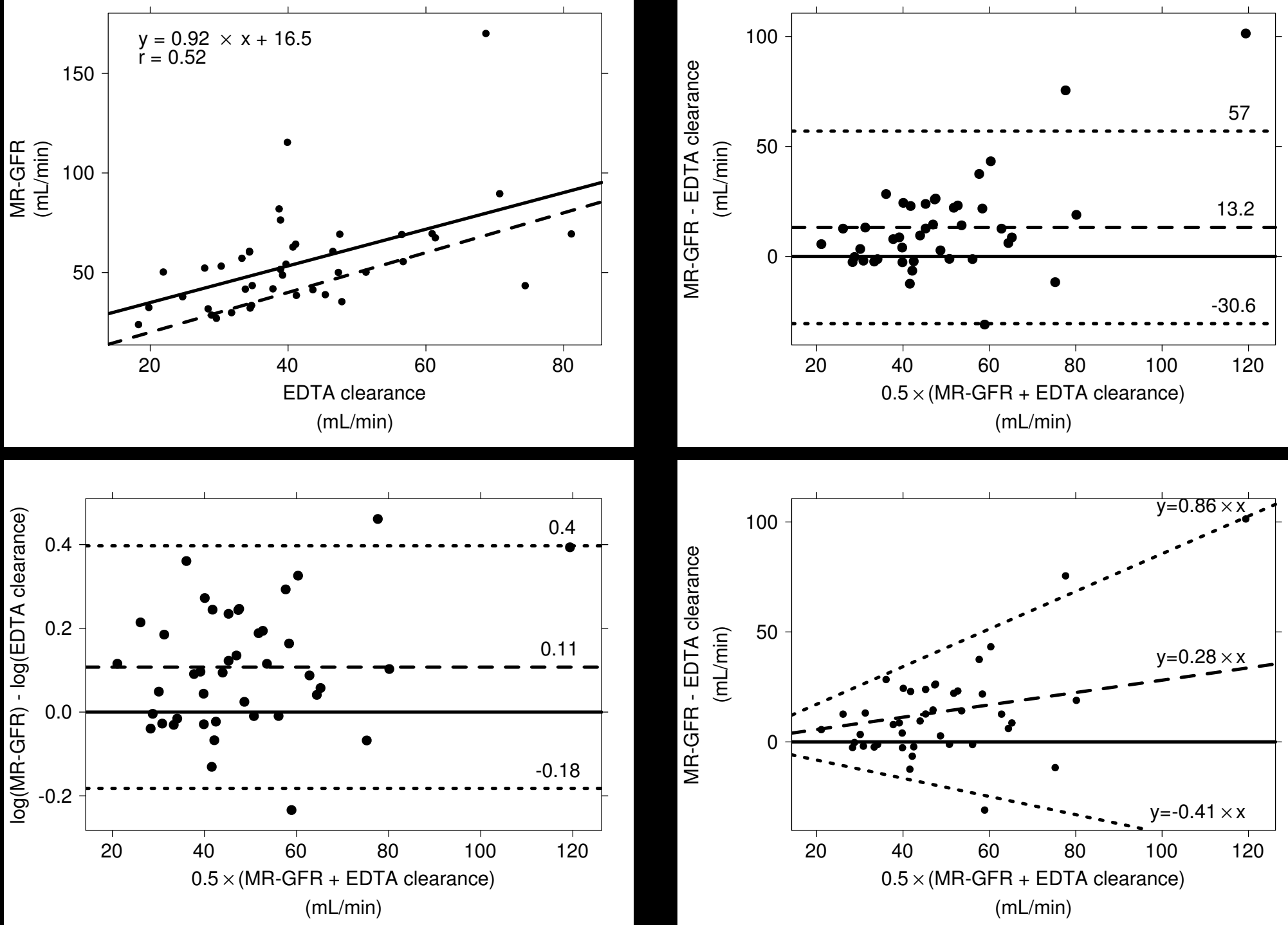

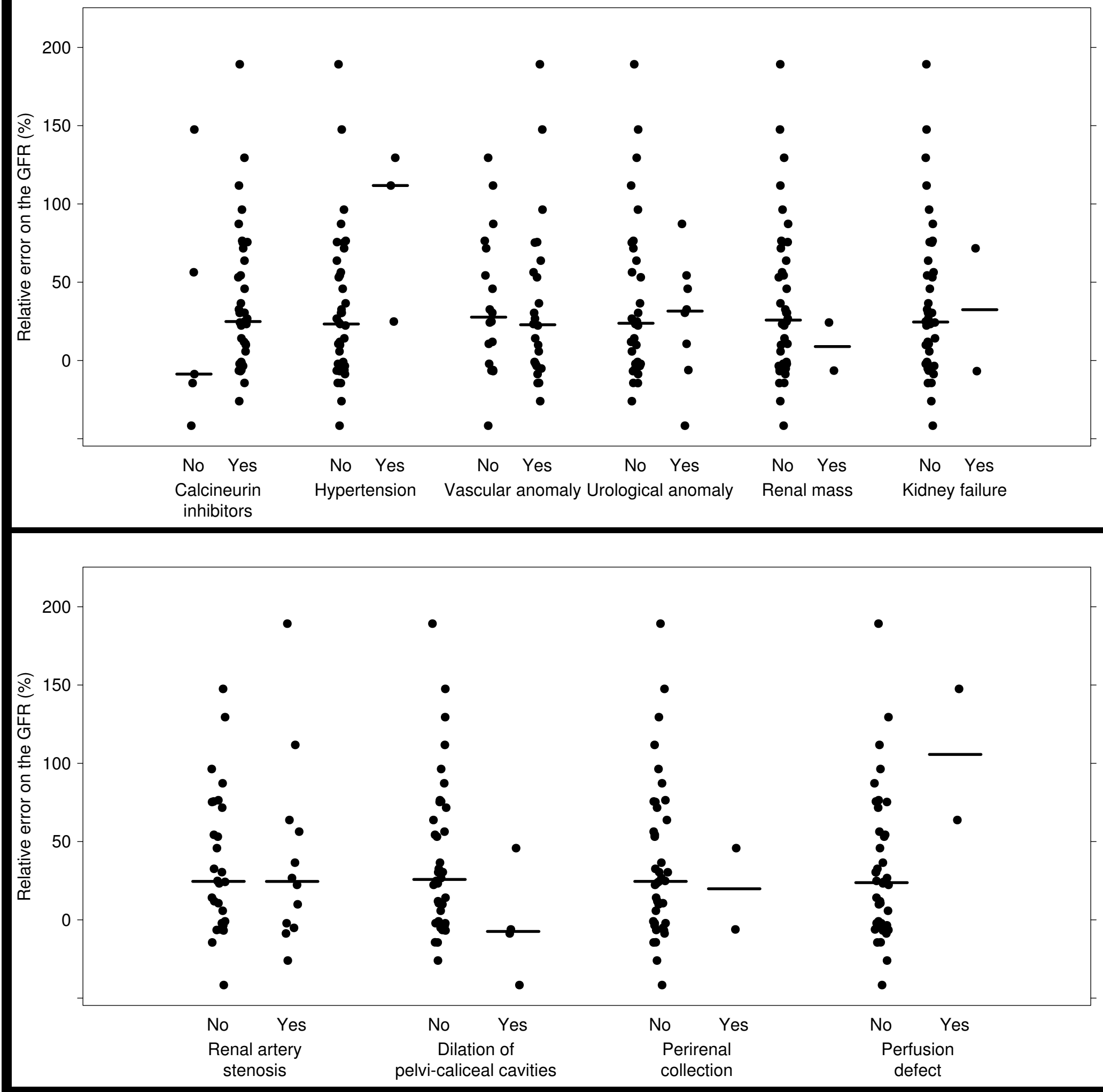
Supplemental material
Click here to download Supplementary File(s): supplemental-material.docx click here to download Supplementary File(s): supplemental-material.docx

\author{
X

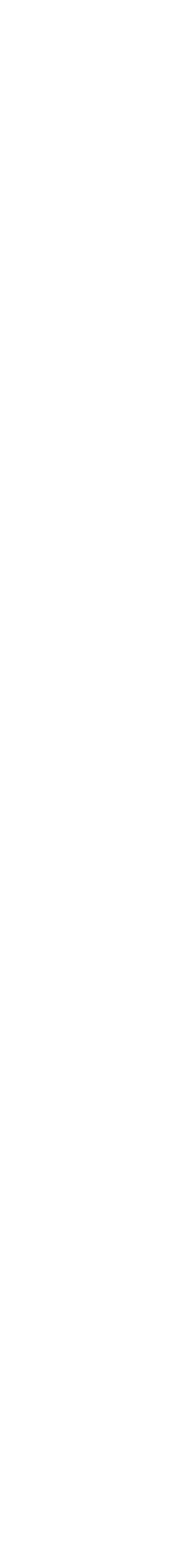

